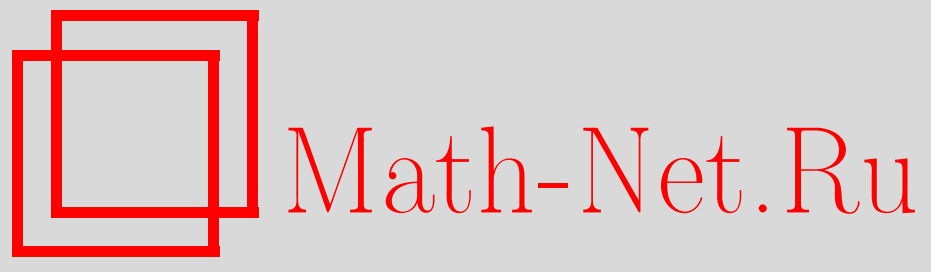

А. А. Владимиров, Замечания о минорантах лапласиана на геометрическом графе, Матем. заметки, 2015, том 98, выпуск 3, 467-469

DOI: https://doi.org/10.4213/mzm10760

Использование Общероссийского математического портала Math-Net.Ru подразумевает, что вы прочитали и согласны с пользовательским соглашением http://www . mathnet.ru/rus/agreement

Параметры загрузки:

IP : 54.210 .77 .194

26 апреля 2023 г., 11:55:36

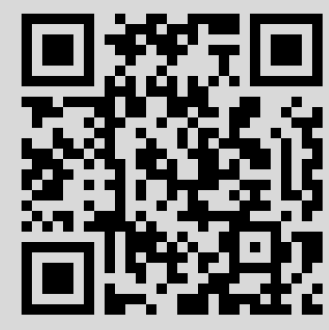




\section{Замечания о минорантах лапласиана на геометрическом графе}

\section{А. А. Владимиров}

1. Геометрическим графом мы далее будем называть совокупность $\Gamma$ из множества $\bigcup_{i=1}^{n}\{i\} \times\left[0, \ell_{i}\right]$ и конечного (возможно, пустого) списка "склеивающих" точки указанного множества равенств вида $\left(i, x_{i}\right)=\left(j, x_{j}\right)$, где $i, j \in 1, \ldots, n, x_{i} \in\left\{0, \ell_{i}\right\}$ и $x_{j} \in\left\{0, \ell_{j}\right\}$. Без существенного ограничения общности рассмотрения мы также будем предполагать выполненным равенство $\sum_{i=1}^{n} \ell_{i}=1$.

Для любой (частично определенной) функции $y: \Gamma \rightarrow \mathbb{R}$ через $y_{i}$ далее будем обозначать соответствующие отображения $y_{i}:\left[0, \ell_{i}\right] \rightarrow \mathbb{R}$ вида $y_{i}(x) \equiv y(i, x)$.

Под суммируемой функцией, определенной почти всюду на $\Gamma$, естественным образом понимается частичное отображение $y: \Gamma \rightarrow \mathbb{R}$, удовлетворяющее соотношениям $y_{i} \in L_{1}\left[0, \ell_{i}\right]$. Интегралом такой функции считается при этом величина

$$
\int_{\Gamma} y d x \rightleftharpoons \sum_{i=1}^{n} \int_{0}^{\ell_{i}} y_{i} d x
$$

Под $W_{2}^{1}(\Gamma)$ далее будем понимать пространство непрерывных функций $y: \Gamma \rightarrow \mathbb{R}$, удовлетворяющих соотношениям $y_{i} \in W_{2}^{1}\left[0, \ell_{i}\right]$. Норма функции $y \in W_{2}^{1}(\Gamma)$ определяется при этом равенством

$$
\|y\|_{W_{2}^{1}(\Gamma)}^{2}=\sum_{i=1}^{n} \int_{0}^{\ell_{i}}\left(\left|y_{i}^{\prime}\right|^{2}+\left|y_{i}\right|^{2}\right) d x .
$$

В случае, когда $\mathfrak{b}$ есть некоторый список вершин графа $\Gamma$, через $W_{2}^{1}(\Gamma, \mathfrak{b})$ будем обозначать подпространство в $W_{2}^{1}(\Gamma)$, выделенное условием $\left.y\right|_{\mathfrak{b}}=0$.

Под $W_{2}^{-1}(\Gamma, \mathfrak{b})$ далее будем понимать пополнение пространства $L_{2}(\Gamma)$ по норме

$$
\|y\|_{W_{2}^{-1}(\Gamma, \mathfrak{b})} \rightleftharpoons \sup _{\|z\|_{W_{2}^{1}(\Gamma, \mathfrak{b})}=1} \int_{\Gamma} y z d x .
$$

Через $\langle y, z\rangle$, где $y=\lim _{n \rightarrow \infty} y_{n} \in W_{2}^{-1}(\Gamma, \mathfrak{b}), y_{n} \in L_{2}(\Gamma)$ и $z \in W_{2}^{1}(\Gamma, \mathfrak{b})$, при этом будет обозначаться предел последовательности интегралов $\int_{\Gamma} y_{n} z d x$.

2. Рассмотрим семейство $P(\Gamma, \mathfrak{b})$ непрерывных функций $y: \Gamma \rightarrow \mathbb{R}$, удовлетворяющих условию $\left.y\right|_{\mathfrak{b}}=0$ и таких, что каждая из функций $y_{i} \in C\left[0, \ell_{i}\right]$ кусочно-линейна. Имеет место следующий простой факт.

УтвеРЖДЕние 2.1. Множество $P(\Gamma, \mathfrak{b})$ плотно в пространстве $W_{2}^{1}(\Gamma, \mathfrak{b})$.

Обозначим для произвольно фиксированной функции $y \in P(\Gamma, \mathfrak{b})$ через $\left\{\zeta_{k}\right\}_{k=0}^{m}$ упорядоченный по возрастанию набор ее значений в точках ее излома (включая в число таковых вершины графа Г). Независимо от выбора индекса $k \in 1, \ldots, m$ полный прообраз интервала $\Lambda_{k} \rightleftharpoons\left(\zeta_{k-1}, \zeta_{k}\right)$ допускает представление

$$
y^{-1}\left(\Lambda_{k}\right)=\bigsqcup_{l=1}^{s_{k}}\left\{i_{k, l}\right\} \times\left(\alpha_{k, l}, \beta_{k, l}\right),
$$

в котором каждый из интервалов $\left(\alpha_{k, l}, \beta_{k, l}\right)$ линейно отображается соответствующей функцией $y_{i_{k, l}}$ на интервал $\Lambda_{k}$. Тем самым, равноизмеримая с $y$ неубывающая функция $Y \in$

DOI: $10.4213 / \mathrm{mzm10760}$ 
$L_{1}[0,1]$ непрерывна и кусочно-линейна, а также удовлетворяет соотношениям

$$
\inf s_{k}^{2} \times \int_{0}^{1}\left|Y^{\prime}\right|^{2} d x=\inf s_{k}^{2} \times \sum_{k=1}^{m} \frac{\left(\zeta_{k}-\zeta_{k-1}\right)^{2}}{\sum_{l=1}^{s_{k}}\left(\beta_{k, l}-\alpha_{k, l}\right)} \leqslant \sum_{k=1}^{m} \sum_{l=1}^{s_{k}} \frac{\left(\zeta_{k}-\zeta_{k-1}\right)^{2}}{\beta_{k, l}-\alpha_{k, l}}=\int_{\Gamma}\left|y^{\prime}\right|^{2} d x .
$$

При этом в случае выполнения условия

$$
\begin{aligned}
& \text { "каждое ребро графа Г может быть включено в бесповторную } \\
& \text { цепь ребер, свлзываюшую некоторые вершины из списка b" }
\end{aligned}
$$

(cp. [1; теорема 1]) заведомо справедлива оценка inf $s_{k} \geqslant 2$.

3. Далее всегда будет предполагаться, что граф Г связен, а список $\mathfrak{b}$ непуст. Следуя методологии работ [2], [3], свяжем с формальной граничной задачей

$$
\left\{\begin{array}{l}
-y^{\prime \prime}=\lambda r y \\
\left.y\right|_{\mathfrak{b}}=0
\end{array}\right.
$$

где $r \in W_{2}^{-1}(\Gamma)$ - некоторая неотрицательная обобщенная функция, линейный операторный пучок $L: \mathbb{R} \rightarrow \mathcal{B}\left(W_{2}^{1}(\Gamma, \mathfrak{b}), W_{2}^{-1}(\Gamma, \mathfrak{b})\right)$ вида

$$
\langle L(\lambda) y, y\rangle \equiv \int_{\Gamma}\left|y^{\prime}\right|^{2} d x-\lambda\left\langle r, y^{2}\right\rangle .
$$

Целью настоящего пункта является нахождение априорной миноранты наименьшего собственного значения пучка $L$ в случае $r=1+m \rho$, где $m \geqslant 0$ - фиксированная постоянная, а $\rho \in W_{2}^{-1}(\Gamma)$ - произвольная неотрицательная обобщенная функция со свойством $\langle\rho, 1\rangle=1$.

Согласно результатам предыдущего пункта независимо от выбора функции $y \in P(\Gamma, \mathfrak{b})$ справедливы соотношения

$$
\begin{aligned}
\langle L(\lambda) y, y\rangle & \geqslant \int_{\Gamma}\left[\left|y^{\prime}\right|^{2}-\lambda y^{2}\right] d x-\lambda m \cdot \sup _{x \in \Gamma} y^{2}(x) \\
& \geqslant \int_{0}^{1}\left[\theta^{2}\left|Y^{\prime}\right|^{2}-\lambda Y^{2}\right] d x-\lambda m \cdot \sup \left\{Y^{2}(0), Y^{2}(1)\right\},
\end{aligned}
$$

где $\theta=1$ в общем случае и $\theta=2$ в случае выполнения условия (1). С учетом утверждения 2.1 и заведомого существования точки $a \in[0,1]$ со свойством $Y(a)=0$, это означает (cp. [4], [5]) неотрицательность оператора $L(\lambda)$ в случае, когда величина $\lambda \in \mathbb{R}$ минорирует наименьшие собственные значения всех граничных задач вида

$$
\left\{\begin{array} { l } 
{ - \theta ^ { 2 } y ^ { \prime \prime } = \lambda ( 1 + m \boldsymbol { \delta } _ { 0 } ) y , } \\
{ y ^ { \prime } ( 0 ) = y ( a ) = 0 , \quad a \in ( 0 , 1 ] , }
\end{array} \quad \left\{\begin{array}{l}
-\theta^{2} y^{\prime \prime}=\lambda\left(1+m \boldsymbol{\delta}_{1}\right) y \\
y(a)=y^{\prime}(1)=0, \quad a \in[0,1) .
\end{array}\right.\right.
$$

Через $\boldsymbol{\delta}_{\zeta} \in W_{2}^{-1}[0,1]$ здесь обозначена дельта-функция Дирака с сосредоточенным в точке $\zeta \in[0,1]$ носителем. Переписывая указанные задачи в эквивалентной форме

$$
\left\{\begin{array} { l } 
{ - \theta ^ { 2 } y ^ { \prime \prime } = \lambda y , } \\
{ y ^ { \prime } ( 0 ) + \frac { \lambda m } { \theta ^ { 2 } } y ( 0 ) = y ( a ) = 0 , \quad a \in ( 0 , 1 ] , }
\end{array} \quad \left\{\begin{array}{l}
-\theta^{2} y^{\prime \prime}=\lambda y, \\
y(a)=y^{\prime}(1)-\frac{\lambda m}{\theta^{2}} y(1)=0, \quad a \in[0,1),
\end{array}\right.\right.
$$

легко устанавливаем, что операторы $L(\lambda)$ неотрицательны при всех $\lambda \leqslant \lambda_{0}$, где через $\lambda_{0}$ обозначено решение уравнения

$$
\frac{\sqrt{\lambda}}{\theta}=\operatorname{arcctg}\left(\frac{m \sqrt{\lambda}}{\theta}\right)
$$

Тем самым, имеет место следующий факт. 
УтвеРЖДЕнИЕ 3.1. Наименъшее собственное значение пучка $L$ с весом $r=1+m \rho$, где $m \geqslant 0$, a $\rho \in W_{2}^{-1}(\Gamma)$ неотрицательна и удовлетворяет равенству $\langle\rho, 1\rangle=1$, минорируется решением уравнения (3).

Утверждение 3.1 представляет собой уточнение и обобщение установленной в [1; п. 5] для $\rho \in W_{2}^{-1}(\Gamma)$ некоторого специального вида оценки наименьшего собственного значения пучка $L$ величиной $\pi^{2} \theta^{2} \cdot(2+2 m)^{-2}$.

4. Аналогичные проведенным в предыдущем пункте рассуждения позволяют оценить снизу также второе собственное значение пучка $L$. А именно, никакое двумерное подпространство линейного множества $P(\Gamma, \mathfrak{b})$ не может не содержать нетривиальной функции $y$ со свойством $\sup _{x \in \Gamma} y(x)+\inf _{x \in \Gamma} y(x)=0$. При этом равноизмеримая с функцией $y \in P(\Gamma, \mathfrak{b})$ неубывающая функция $Y \in W_{2}^{1}[0,1]$ удовлетворяет равенству $Y(0)+Y(1)=0$. Вместе с соотношениями (2) и утверждением 2.1 это означает, что квадратичная форма оператора $L(\lambda)$ не может быть отрицательно определена на каком-либо двумерном подпространстве пространства $W_{2}^{1}(\Gamma, \mathfrak{b})$, если справедливо неравенство $\lambda \leqslant \lambda_{0}$, где через $\lambda_{0}$ обозначено наименьшее собственное значение граничной задачи

$$
\left\{\begin{array}{l}
-\theta^{2} y^{\prime \prime}=\lambda\left(1+m \boldsymbol{\delta}_{1}\right) y, \\
y(0)+y(1)=y^{\prime}(0)+y^{\prime}(1)=0 .
\end{array}\right.
$$

Тем самым, справедливо следующее утверждение.

УтверЖдЕние 4.1. Второе собственное значение пучка $L$ с весом $r=1+m \rho$, где $m \geqslant$ 0, a $\rho \in W_{2}^{-1}(\Gamma)$ неотрицательна и удовлетворяет равенству $\langle\rho, 1\rangle=1$, минорируется решением уравнения

$$
\frac{\sqrt{\lambda}}{[2 \theta]}=\operatorname{arcctg}\left(\frac{m \sqrt{\lambda}}{[2 \theta]}\right) \text {. }
$$

\section{СПИСОК ЦИТИРОВАННОЙ ЛИТЕРАТУРЫ}

[1] А. Т. Диаб, П.А. Кулешов, О.М. Пенкин, Матем. заметки, 96:6 (2014), 885-895. [2] М. И. Нейман-заде, А. А. Шкаликов, Матем. заметки, 66:5 (1999), 723-733. [3] А. А. Владимиров, Матем. заметки, 75:6 (2004), 941-943. [4] Ю. В. Егоров, В. А. Кондратьев, УМH, 51:3 (1996), 73-144. [5] E.S. Karulina, A. A. Vladimirov, Tatra Mt. Math. Publ., 54 (2013), 101-118.

\section{А. А. Владимиров}

Вычислительный центр им. А.А. Дородницына

Российской академии наук, г. Москва

E-mail: vladimi@mech.math.msu.su
Поступило

15.04 .2015 